\section{Cobertura da Comunicação de Informação Hospitalar e Ambulatorial (CIHA) utilizando os partos registrados no Sistema de Informações sobre Nascidos Vivos, Brasil, 2006 a 2009}

\author{
Coverage of the Hospital and Outpatient \\ Information Form (CIHA) using births recorded in \\ the Information System on Live Births (SINASC), \\ Brazil, 2006-2009
}

Rejane Sobrino Pinheiro 1,2

Juliana Romualdo do Nascimento da Silva ${ }^{3}$ Cláudia Risso de Araujo Lima 4

Cláudia Medina Coeli 1,2

\footnotetext{
1 Instituto de Estudos em Saúde Coletiva, Universidade Federal do Rio de Janeiro, Rio de Janeiro, Brasil.

2 Faculdade de Medicina, Universidade Federal do Rio de Janeiro, Rio de Janeiro, Brasil.

${ }^{3}$ Subsecretaria de Vigilância em Saúde, Secretaria Estadual de Saúde do Rio de Janeiro, Rio de Janeiro, Brasil. 4 Coordenação Geral de Disseminação de Informações em Saúde, Ministério da Saúde, Rio de Janeiro, Brasil.

Correspondência R. S. Pinheiro Instituto de Estudos em Saúde Coletiva, Universidade Federal do Rio de Janeiro. Av. Horácio Macedo s/n, Cidade Universitária, Rio de Janeiro. RJ 21941-598, Brasil. rejane@iesc.ufrj.br
}

\section{Abstract}

The Brazilian Ministry of Health established mandatory completion of the Hospital and Outpatient Information Form (CIHA) in all the country's health services in order to monitor care funded by sources other than the Unified National Health System (SUS). Considering that data quality is essential, this study analyzed coverage of the CIHA in the States and Federal District, taking data from the Information System on Live Births (SINASC) from 2006 to 2009 as the standard. Coverage of the CIHA was low in nearly all of the States, corresponding to fewer than one-fourth of the births recorded in the SINASC, decreasing from 24.4\% in 2006 to 19.7\% in 2009. There was a wide variation between the States, with the best results in São Paulo (71.9\% in 2006; $46.4 \%$ in 2009), Rio Grande do Sul (35.8\% in 2006; 29.5\% in 2009), and Santa Catarina (31.6\% in 2006; 37.7\% in 2009). The other States showed less than 20\% coverage. Mechanisms for data collection and use of the CIHA to support decisionmaking are important for planning and evaluating healthcare.

Supplemental Health; Underregistration; Hospital Information System; Birth Certificates

\section{Introdução}

No ano de 2005, 20\% da população brasileira possuíam algum plano de saúde privado e, em 2008, a cobertura chegou a $25,9 \%$, dada a tendência crescente do número de beneficiários 1. Em 2009, o Brasil contava com cerca de 42 milhões de beneficiários de planos de saúde, e apenas $2 \%$ dos municípios não possuíam população coberta ${ }^{2}$.

Esses dados indicam que, para uma melhor compreensão da situação de saúde da população brasileira, é necessário que se obtenham informações tanto do setor público quanto do setor da saúde suplementar.

Para acompanhamento da assistência prestada à população assistida com financiamento não SUS, foi instituída pelo Ministério da Saúde, em março de 1999, a obrigatoriedade do preenchimento da Comunicação de Internação Hospitalar (CIH), para todos os estabelecimentos privados situados no território nacional ${ }^{3}$. A CIH passa, então, a ser instrumento obrigatório de informação dos eventos de internação realizados sem o financiamento do SUS, a serem encaminhadas pelos estabelecimentos de saúde às Secretarias Municipais/Estaduais de Saúde, que têm a responsabilidade de transmissão ao Ministério da Saúde. Em outubro de 2005, é instituída, no âmbito da Agência Nacional de Saúde Suplementar (ANS), a CIH para acompanhamento da assistência prestada aos beneficiários de planos de saúde 4 . 
Desde a sua criação, a estrutura da CIH sofreu alterações e mudou sua denominação para Comunicação de Informação Hospitalar e Ambulatorial (CIHA), sendo constituída, atualmente, por informações sobre o estabelecimento de saúde, o usuário, o evento e o financiamento da internação e do atendimento ambulatorial 5,6.

O aprimoramento da qualidade dos dados do sistema de informação em saúde do país, do qual a CIHA faz parte, é indispensável para uma melhor gestão do sistema de saúde e consequente melhoria da situação de saúde da população brasileira 7 .

Uma importante dimensão de qualidade de uma base de dados no apoio à gestão é a sua cobertura. A avaliação e a melhoria da qualidade de um conjunto de dados podem ser realizadas com base na informação registrada em outras bases de dados. O Sistema de Informações sobre Nascidos Vivos (SINASC) tem como objetivo registrar todos os partos de nascidos vivos no país, tanto os financiados pelo SUS quanto os com financiamento privado. O SINASC é um sistema de abrangência nacional com cobertura considerada elevada, acima de $90 \%$ em diversas regiões do país 8,9 , que pode ser considerado uma referência para a estimativa da cobertura da CIHA no território nacional, com base na comparação dos dados nela registrados.

Considerando que a apropriação das informações pelos gestores e pela sociedade organizada para subsidiar as suas atividades depende, dentre outros fatores, da credibilidade da qualidade dos dados, este estudo se propõe analisar a cobertura da CIHA entre as Unidades da Federação (UF) do Brasil, tomando como fonte de comparação os dados do SINASC.

\section{Método}

Para estimar o número de partos financiados pelo setor privado realizados em hospitais, foram utilizados os dados do SINASC e os do Sistema de Informações Hospitalares do SUS (SIH-SUS), no período de janeiro de 2006 a setembro de 2009, cujo algoritmo está apresentado no diagrama da Figura 1.

Em uma primeira etapa, utilizou-se o Cadastro Nacional de Estabelecimentos de Saúde (CNES) para identificar as unidades de saúde pública.

Em seguida, obteve-se, no SIH-SUS, a quantidade, por UF, de partos realizados em estabelecimentos de saúde não públicos (filantrópico, privado e universitário) pagos com financiamento do SUS.
No próximo passo, foram eliminados do SINASC todos os registros de nascidos vivos de partos não hospitalares, os registros de partos realizados em hospitais sob regime público (hospital federal, estadual ou municipal) e os registros de nascidos vivos de partos múltiplos, permanecendo apenas um. Da quantidade restante de nascidos vivos por UF, foi subtraída a quantidade de partos realizados em hospitais não públicos pagos com financiamento do SUS. Com isso, obteve-se do SINASC o número de nascidos vivos dos partos realizados em hospitais privados sem financiamento do SUS, por UF.

Para obter na base de dados da CIHA os partos realizados em estabelecimentos privados e não pagos pelo SUS, foram excluídos os registros de partos cujo código da fonte pagadora não fosse: convênio-plano privado; particular-pessoa física; convênio-plano público e particular-pessoa jurídica (códigos 1, 2, 6 e 9, respectivamente).

A cobertura de partos da CIHA foi calculada com o percentual correspondente ao total de partos na CIHA em relação a total de partos em hospitais privados sem financiamento do SUS registrados no SINASC, segundo UF, obtido de etapa anterior.

\section{Resultados}

Observou-se que a cobertura da CIHA foi baixa em praticamente todas as UF em todo o período analisado, registrando menos de um quarto dos partos constantes no SINASC (Tabela 1). A cobertura decresceu desde 2006 (24,4\%), ano posterior à portaria que altera o formulário de coleta, até 2009 (19,7\%).

A variação entre as UF foi bastante expressiva, sendo São Paulo (71,9\% em 2006; 46,4\% em 2009), Rio Grande do Sul (35,8\% em 2006; 29,5\% em 2009) e Santa Catarina (31,6\% em 2006; 37,7\% em 2009) os que apresentaram os melhores resultados no período. Todas as outras UF apresentaram cobertura inferior a $20 \%$.

Na Região Norte, com exceção do Acre, todos os estados apresentaram índices de cobertura dos partos menores que 4\%. No Amapá e em Tocantins, não houve registro de dados da CIHA para quaisquer procedimentos, e houve apenas 63 procedimentos no ano de 2006 (oito partos) no Amazonas.

Na Região Nordeste, destacou-se o Ceará $(9,8 \%$ em $2006 ; 15,8 \%$ em 2009$)$, seguido de Sergipe $(7,9 \%$ em 2006; 18,6\% em 2009), com crescimento da cobertura em ambos os estados. A Bahia, terceiro melhor valor da região, apresentou cobertura $6,5 \%$ em 2006 , reduzindo para 
Fluxograma da identificação do número de partos sem financiamento do SUS: $A=$ número de partos realizados em hospitais não-públicos com vínculos com o SUS; B = número de partos em hospitais não-públicos, Brasil, 2006 a 2009.

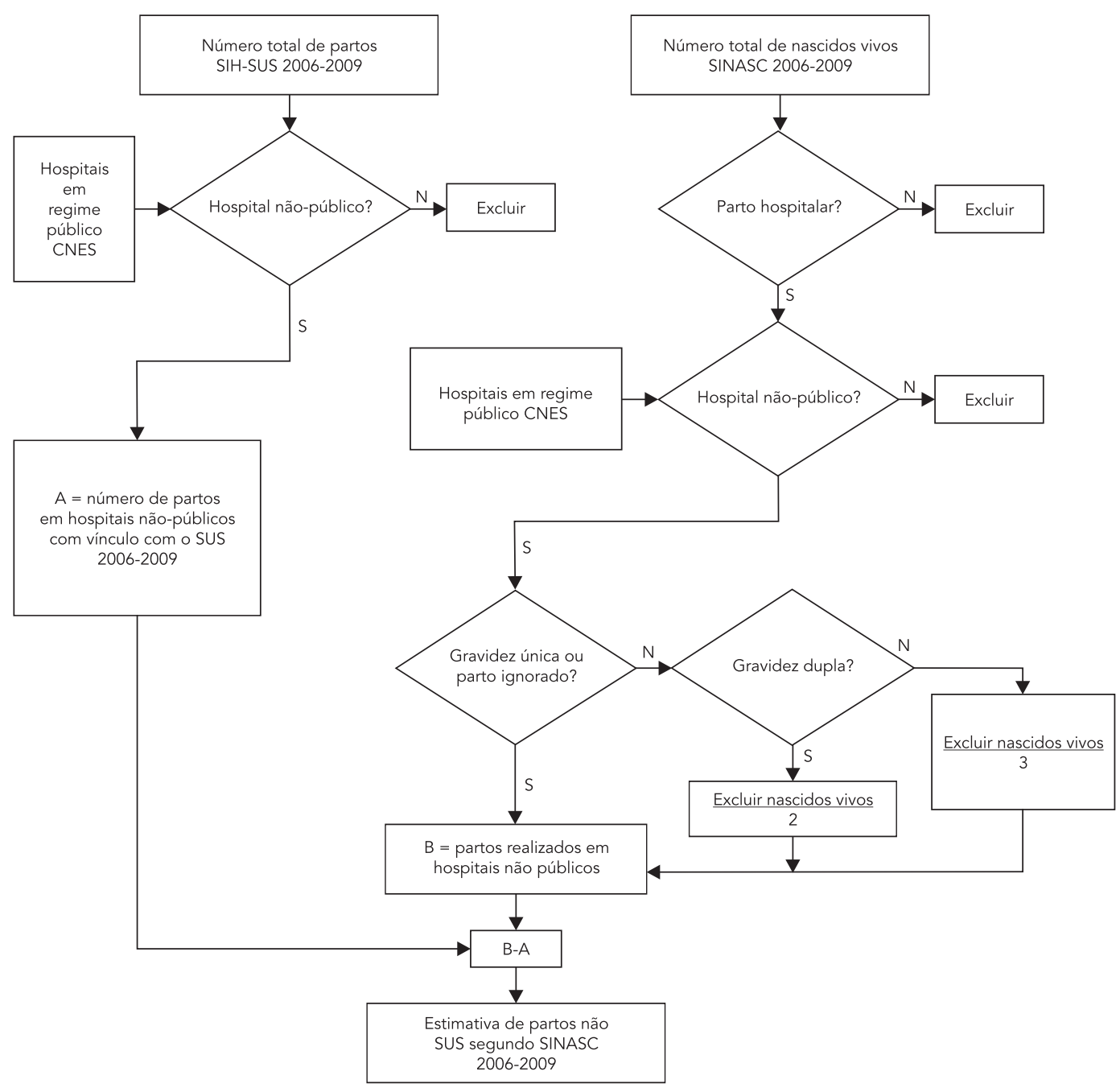

CNES: Cadastro Nacional de Estabelecimentos de Saúde; SIH-SUS: Sistema de Informações Hospitalares do SUS;

SINASC: Sistema de Informações sobre Nascidos Vivos.

3,4\% em 2009. Os demais estados apresentaram índices decrescentes, a maior parte deles abaixo de $1,5 \%$.

Na Região Centro-oeste, as melhores coberturas foram em Mato Grosso e Mato Grosso do Sul, porém nenhum índice de cobertura atingiu $14 \%$. Enquanto no primeiro estado houve tendência de crescimento, no outro houve progressivo decréscimo, sendo a cobertura em 2009 cerca da metade da encontrada em 2006. Goiás apresentou forte declínio da cobertura, ao passo que o Distrito Federal mostrou instabilidade no período.

Na Região Sudeste, os índices foram decrescentes para todos os estados. Enquanto Espírito Santo e Minas Gerais tiveram cobertura de partos variando entre $10 \%$ a $18 \%$ no período, o maior contraste foi observado no Rio de Janeiro e São Paulo.

O Estado de São Paulo foi o que apresentou a maior cobertura dos partos no país. Já o Rio de Janeiro mostrou índices bem abaixo dos esperados 
Número de partos segundo Unidade da Federação (UF), Brasil, 2006 a setembro de 2009.

\begin{tabular}{|c|c|c|c|c|c|c|c|c|c|c|c|c|}
\hline \multirow[t]{2}{*}{ UF } & \multicolumn{3}{|c|}{2006} & \multicolumn{3}{|c|}{2007} & \multicolumn{3}{|c|}{2008} & \multicolumn{3}{|c|}{2009} \\
\hline & SINASC * & $\mathrm{CIHA} * *$ & $\begin{array}{l}\text { Cober- } \\
\text { tura (\%) }\end{array}$ & SINASC * & $\mathrm{CIHA} * \star$ & $\begin{array}{l}\text { Cober- } \\
\text { tura (\%) }\end{array}$ & SINASC * & $\mathrm{CIHA} * \star$ & $\begin{array}{l}\text { Cober- } \\
\text { tura (\%) }\end{array}$ & SINASC * & $\mathrm{CIHA} * \star$ & $\begin{array}{l}\text { Cober- } \\
\text { tura (\%) }\end{array}$ \\
\hline Região Norte & 42.300 & 1.249 & 3,0 & 45.375 & 1.308 & 2,9 & 64.756 & 1.241 & 1,9 & 43.870 & 380 & 0,9 \\
\hline Rondônia & 8.733 & 33 & 0,4 & 6.982 & 52 & 0,7 & 11.547 & 283 & 2,5 & 9.176 & - & - \\
\hline Acre & 1.913 & 319 & 16,7 & 1.303 & 625 & 48,0 & 3.027 & 333 & 11,0 & 2.020 & 18 & 0,9 \\
\hline Amazonas & 7.153 & 8 & 0,1 & 11.288 & - & - & 19.030 & - & - & 14.637 & - & - \\
\hline Roraima & 2.102 & 76 & 3,6 & 3.200 & 14 & 0,4 & 2.609 & - & - & 1.014 & - & - \\
\hline Pará & 15.855 & 813 & - & 15.632 & 617 & 3,9 & 20.747 & 625 & 3,0 & 13.761 & 362 & 2,6 \\
\hline Amapá & 2.018 & - & - & 2.700 & - & - & 3.220 & - & - & 574 & - & - \\
\hline Tocantins & 4.526 & - & 18,0 & 4.270 & - & - & 4.577 & - & - & 2.689 & - & - \\
\hline Região Nordeste & 120.149 & 4.870 & 4,1 & 136.030 & 4.255 & 3,1 & 178.037 & 6.292 & 3,5 & 104.842 & 4.549 & 4,3 \\
\hline Maranhão & 25.303 & 89 & 0,4 & 26.088 & 305 & 1,2 & 29.152 & 269 & 0,9 & 16.866 & 210 & 1,2 \\
\hline Piauí & 3.214 & 69 & 2,1 & 7.965 & 54 & 0,7 & 5.856 & - & - & 4.614 & - & - \\
\hline Ceará & 14.161 & 1.392 & 9,8 & 13.531 & 1.540 & 11,4 & 25.630 & 3.310 & 12,9 & 14.412 & 2.283 & 15,8 \\
\hline Rio Grande do Norte & 2.714 & 25 & 0,9 & 6.201 & 32 & 0,5 & 7.583 & - & - & 5.611 & 32 & 0,6 \\
\hline Paraíba & 9.471 & 196 & 2,1 & 11.208 & 13 & 0,1 & 13.741 & 1.005 & 7,3 & 7.777 & 542 & 7,0 \\
\hline Pernambuco & 31.047 & 1.142 & 3,7 & 36.213 & 131 & 0,4 & 33.572 & 50 & 0,1 & 20.075 & 4 & 0,0 \\
\hline Alagoas & 5.852 & 48 & 0,8 & 6.327 & 17 & 0,3 & 8.706 & - & - & 5.596 & - & - \\
\hline Sergipe & 3.871 & 304 & 7,9 & 4.901 & 208 & 4,2 & 6.393 & 654 & 10,2 & 3.035 & 565 & 18,6 \\
\hline Bahia & 24.516 & 1.605 & 6,5 & 23.596 & 1.955 & 8,3 & 47.405 & 1.004 & 2,1 & 26.855 & 913 & 3,4 \\
\hline Região Sudeste & 313.691 & 119.227 & 38,0 & 391.211 & 95.153 & 24,3 & 458.559 & 113.731 & 24,8 & 320.645 & 92.115 & 28,7 \\
\hline Minas Gerais & 54.748 & 8.731 & 15,9 & 57.821 & 5.919 & 10,2 & 64.888 & 6.770 & 10,4 & 44.561 & 5.436 & 12,2 \\
\hline Espírito Santo & 14.176 & 2.465 & 17,4 & 13.868 & 1.769 & 12,8 & 19.625 & 2.207 & 11,2 & 12.380 & 1.743 & 14,1 \\
\hline Rio de Janeiro & 98.598 & 2.907 & 2,9 & 93.905 & 2.723 & 2,9 & 115.096 & 2.414 & 2,1 & 83.591 & 1.401 & 1,7 \\
\hline São Paulo & 146.169 & 105.124 & 71,9 & 225.617 & 84.742 & 37,6 & 258.950 & 102.340 & 39,5 & 180.112 & 83.535 & 46,4 \\
\hline Região Sul & 120.578 & 30.352 & 25,2 & 116.114 & 25.776 & 22,2 & 134.390 & 22.987 & 17,1 & 94.713 & 22.533 & 23,8 \\
\hline Paraná & 51.184 & 6.549 & 12,8 & 50.012 & 6.187 & 12,4 & 59.881 & 5.964 & 10,0 & 41.891 & 5.371 & 12,8 \\
\hline Santa Catarina & 24.994 & 7.901 & 31,6 & 23.761 & 6.908 & 29,1 & 26.700 & 7.890 & 29,6 & 19.338 & 7.288 & 37,7 \\
\hline Rio Grande do Sul & 44.400 & 15.902 & 35,8 & 42.341 & 12.681 & 29,9 & 47.809 & 9.133 & 19,1 & 33.484 & 9.874 & 29,5 \\
\hline Região Centro-oeste & 64.077 & 5.228 & 8,2 & 68.153 & 3.169 & 4,6 & 73.647 & 4.407 & 6,0 & 52.915 & 2.139 & 4,0 \\
\hline Mato Grosso do Sul & 7.336 & 995 & 13,6 & 10.759 & 798 & 7,4 & 11.217 & 823 & 7,3 & 7.740 & 521 & 6,7 \\
\hline Mato Grosso & 12.423 & 903 & 7,3 & 15.121 & 1.045 & 6,9 & 15.051 & 1.652 & 11,0 & 9.811 & 1.118 & 11,4 \\
\hline Goiás & 41.535 & 3.248 & 7,8 & 40.997 & 1.326 & 3,2 & 44.633 & 712 & 1,6 & 34.143 & 500 & 1,5 \\
\hline Distrito Federal & 2.783 & 82 & 2,9 & 1.276 & - & - & 2.747 & 1.220 & 44,4 & 1.221 & - & - \\
\hline Total & 660.795 & 160.926 & 24,4 & 756.883 & 129.661 & 17,1 & 909.387 & 148.658 & 16,3 & 616.983 & 121.716 & 19,7 \\
\hline
\end{tabular}

CIHA: Comunicação de Informação Hospitalar e Ambulatorial; CNES: Cadastro Nacional de Estabelecimentos de Saúde; SIH-SUS: Sistema de Informações Hospitalares do Sistema Único de Saúde; SINASC: Sistema de Informações sobre Nascidos Vivos.

* Partos sem financiamento do SUS;

** Fonte de financiamento convênio-plano privado, particular-pessoa física, convênio-plano público e particular-pessoa jurídica.

para um dos principais estados do país: 2,9\% em 2006 e $1,7 \%$ em 2009.

Na Região Sul, Santa Catarina e Rio Grande do Sul apresentaram cobertura dos partos em torno de $30 \%$, mantendo certa estabilidade dos índices no período. O Paraná apresentou os menores valores da região, com aproximadamente $12 \%$ de cobertura no quadriênio.

\section{Discussão}

Verificou-se baixa cobertura dos partos da CIHA ao longo do período em praticamente todas as UF. São Paulo foi a que apresentou a maior cobertura, o dobro da segunda maior. A Região Sul, de um modo geral, apresentou coberturas em torno de $30 \%$. As UF das regiões Norte e 
Nordeste apresentaram as menores coberturas, abaixo de $5 \%$.

Benevides 10 analisou a cobertura da CIHA por meio da proporção de hospitais que informaram ter realizado pelo menos uma internação nos anos de 2007 e 2008. Considerou críticas as coberturas abaixo de $31 \%$, o que foi observado para a maioria das UF. Os maiores valores de cobertura foram encontrados em algumas UF das regiões Sudeste e Sul, semelhante ao constatado no presente estudo, e em uma UF da Região Norte (Acre) e em uma da Região Nordeste (Sergipe). Observou que a CIHA possuiu certa regularidade na série histórica mensal de envio de dados dos estabelecimentos de saúde. Os resultados observados pelo autor foram sistematicamente superiores aos verificados no presente estudo, indicando que a maioria dos hospitais não informa a totalidade das internações realizadas.

Ao contrário do que se observa normalmente com a implantação de um sistema de informação, a cobertura reduziu com o passar do tempo, sendo menor em 2009 que em 2006. O mesmo ocorreu nas análises sobre a cobertura de dados por meio dos hospitais informantes no Estado de São Paulo 10,11. Moreira 11 observou, para o Estado de São Paulo, alto grau de consistência no preenchimento da CIHA, acima de $99,5 \%$, pela aplicação da rotina de consistência de dados utilizada pelo Departamento de Informática do SUS (DATASUS), e encontrou cobertura de aproximadamente $93 \%$ das unidades hospitalares em 2002, o dobro do encontrado por Benevides $10 \mathrm{em}$ 2007 e 2008.

Destacam-se duas hipóteses para explicar a redução da cobertura da CIHA no período: falta de informação por parte do prestador e não envio da informação fornecida pelos prestadores aos gestores municipais ou estaduais, responsáveis pela transmissão desses dados ao nível central 5,12. O Ministério da Saúde tem empenhado esforços para a completa implantação da CIHA após sua criação 4: elaboração de programas informatizados gratuitos para preenchimento dos formulários 12; definição de fluxos de envio dos dados para as Secretarias Municipais ou Estaduais de Saúde 12; adequação e aperfeiçoamento dos campos e da tabela de procedimentos da CIHA 6,13,14,15,16; vinculação do cadastro no CNES da unidade prestadora de serviços ao repasse de recursos para as que são integrantes do SUS 13; cobrança na renovação do alvará de funcionamento da Vigilância Sanitária 4; na tramitação de qualquer solicitação ao Ministério 4; e na concessão ou renovação do Certificado de Entidade Beneficente de Assistência Social 17. No entanto, com o passar do tempo, sem a supervisão da informação coletada e o uso inci- piente desse dado na gestão, a cobertura tende a reduzir.

A ANS é uma grande usuária da CIHA 5 e tem apoiado fortemente o DATASUS na definição de melhorias do sistema, a fim de aprimorar a qualidade dos dados. No ano de 2006, foram incorporadas diversas críticas de consistência aos programas de entrada e de consolidação dos dados.

Com abrangência nacional, tal sistema permite obter dados sobre a rede assistencial privada, a epidemiologia da população e as práticas clínicas, além do monitoramento da rede assistencial informada pelas Operadoras de Plano de Saúde 5 . Portanto, a CIHA pode vir a constituir um instrumento valioso para o planejamento, a avaliação da qualidade da assistência, a distribuição geográfica da oferta e a produção dos serviços de saúde, além de produzir dados para estudos sobre a variação do uso de recursos hospitalares, da morbidade e da mortalidade hospitalar.

É importante avaliar a necessidade de utilizar mecanismos de incorporação da qualidade da CIHA nos processos de credenciamento dos prestadores privados de serviços.

Algumas limitações da estratégia de análise adotadas devem ser consideradas. O SINASC apresenta subenumeração de nascidos vivos, em especial nas regiões Norte e Nordeste 8 ,9, e não foram computados os partos dos natimortos, que pode ter levado a uma superestimação da cobertura da CIHA. Por outro lado, também se espera que alguns partos financiados pelo SUS não estejam registrados no SIH-SUS, o que levaria a uma subestimação da cobertura. Entretanto, além dos dois problemas tenderem a se anular, as diferenças nas quantidades de partos registrados no SINASC e na CIHA foi de tal magnitude, que as limitações acima apontadas dificilmente poderiam explicar as baixas coberturas verificadas.

Os resultados do estudo apontam para uma baixa cobertura da CIHA, sugerindo que iniciativas devam ser tomadas com intuito de melhorar a qualidade desse sistema de informação. $\mathrm{O}$ algoritmo proposto no presente estudo representa uma ferramenta útil no acompanhamento da cobertura da CIHA, em função de sua simplicidade e do emprego de bases de dados amplamente disponíveis, como o SINASC e o SIH-SUS. Isso minimiza o custo de recuperação de dados e de processamento, permitindo avaliações iniciais oportunas, em especial se comparado com as alternativas mais acuradas para avaliar cobertura, como os inquéritos. 


\section{Resumo}

Para monitorar a assistência com financiamento não SUS, o Ministério da Saúde do Brasil instituiu a obrigatoriedade do preenchimento da Comunicação de Informação Hospitalar e Ambulatorial (CIHA) em todos os serviços de saúde do país. Considerando que a qualidade dos dados é fundamental, este estudo analisou a cobertura da CIHA nas Unidades da Federação (UF), tomando como padrão os dados do Sistema de Informações sobre Nascidos Vivos (SINASC) do período de 2006 a 2009. A cobertura da CIHA foi baixa em quase todas as UF, menos de um quarto dos partos que foram registrados no SINASC, decrescendo desde 2006 (24,4\%) até 2009 (19,7\%). A variação entre as UF foi expressiva, sendo São Paulo (71,9\% em 2006; 46,4\% em 2009), Rio Grande do Sul (35,8\% em 2006; 29,5\% em 2009) e Santa Catarina (31,6\% em 2006; 37,7\% em 2009) as que apresentaram os melhores resultados. As outras UF apresentaram cobertura inferior a $20 \%$. Mecanismos de supervisão da coleta e o uso da CIHA para apoiar a decisão são atividades importantes na sua constituição como um instrumento para planejamento e avaliação da assistência.

Saúde Suplementar; Sub-registro; Sistemas de Informação Hospitalar; Declaração de Nascimento

\section{Colaboradores}

R. S. Pinheiro, J. R. N. Silva e C. R. A. Lima participaram da análise dos dados, elaboração e revisão crítica do manuscrito. C. M. Coeli participou da elaboração e revisão crítica do manuscrito.

\section{Agradecimentos}

Ao Conselho Nacional de Desenvolvimento Científico e Tecnológico (CNPq) pelo apoio financeiro para a pesquisa.

\section{Referências}

1. Instituto Brasileiro de Geografia e Estatística. Pesquisa Nacional por Amostra de Domicílios: um panorama da saúde no Brasil. Acesso e utilização dos serviços, condição de saúde, e fatores de risco e proteção à saúde, 2008. http://www.ibge.gov.br/ home/estatistica/populacao/panorama_saude_ brasil_2003_2008/PNAD_2008_saude.pdf (acessado em 10/Jan/2011).

2. Agencia Nacional de Saúde Suplementar. Caderno de Informação de Saúde Suplementar: beneficiários, operadoras e planos. Ano 1. Rio de Janeiro: Agência Nacional de Saúde Suplementar; 2007.

3. Ministério da Saúde. Portaria nº ${ }^{\circ} 221$, de 24 de março de 1999. Determina que todas as unidades hospitalares situadas no território nacional, públicas e privadas, passem a informar a ocorrência de todos os eventos de internação hospitalar, independente da fonte de remuneração dos serviços prestados. Diário Oficial da União 1999; 25 mar.
4. Agência Nacional de Saúde Suplementar. Resolução normativa $n^{\circ}$. 113/ANSS, de 13 de outubro de 2005. Institui a utilização da Comunicação de Internação Hospitalar - CIH, no âmbito da ANS para acompanhamento da assistência prestada aos beneficiários de planos privados de assistência à saúde. Diário Oficial de União 2005; 14 out.

5. Ministério da Saúde. Portaria no ${ }^{\circ}$ 1002, de 20 de dezembro de 2002. Altera o layout da CIH - Comunicação de Internação Hospitalar. Diário Oficial da União 2002; 23 dez.

6. Ministério da Saúde. Portaria $n^{\circ}$. 637, de 21 de março de 2007. Altera a estrutura da Comunicação de Internação Hospitalar - CIH. Diário Oficial da União 2007; 22 mar.

7. Rede Interagencial de Informações para a Saúde. Indicadores básicos de saúde no Brasil: conceitos e aplicações. http://www.datasus.gov.br (acessado em 20/Jul/2010). 
8. Barbosa LM, Melo GHN. Avaliação da qualidade das informações sobre fecundidade provenientes do SINASC no Nordeste, 2000. Rev Bras Estud Popul 2005; 22:141-58.

9. Mello-Jorge MHP, Laurenti R, Gotlieb SLD. Análise da qualidade das estatísticas vitais brasileiras: a experiência de implantação do SIM e do SINASC. Ciênc Saúde Coletiva 2007; 12:643-54.

10. Benevides PRS. Sistema de Comunicação de Internação Hospitalar (CIH/SAS/MS): proposta de critérios de avaliação da qualidade das informações [Dissertação de Mestrado]. Rio de Janeiro: Fundação Oswaldo Cruz; 2009.

11. Moreira ML. Cobertura e utilização de serviços de saúde suplementar no Estado de São Paulo [Dissertação de Mestrado]. Rio de Janeiro: Fundação Oswaldo Cruz; 2004.

12. Ministério da Saúde. Portaria $n^{\circ}$. 213, de 11 de maio de 1999. Cria o programa SISCIHOI. Diário Oficial da União 1999; 12 mai.

13. Ministério da Saúde. Portaria nº. 988, de 27 de junho de 2005. Altera a estrutura da Comunicação de Internação Hospitalar. Diário Oficial da União 2005; 28 jun.
14. Ministério da Saúde. Portaria no ${ }^{\circ}$ 1722, de 22 de setembro de 2005. Altera a estrutura da Comunicação de Internação Hospitalar e dá outras providências. Diário Oficial da União 2005; 23 set.

15. Ministério da Saúde. Portaria $n^{\circ}$. 147, de 13 de março de 2008. Alterar, na forma do Anexo desta Portaria, o layout da Comunicação de Internação Hospitalar - CIH de que trata o Anexo I da Portaria $n^{\circ}$. 24/GM, de 10 de janeiro de 2008. Diário Oficial da União 2008; 14 mar.

16. Ministério da Saúde. Portaria no ${ }^{\circ}$ 24, de 10 de janeiro de 2008. Inclui no Sistema de Comunicação de Internação Hospitalar - CIH códigos para registrar os procedimentos que não têm correspondência na tabela SUS. Diário Oficial da União 2008; 11 jan.

17. Presidência da República. Decreto no ${ }^{\circ} .7 .237$, de 20 de julho de 2010. Diário Oficial de União 2010; 21 jul.

Recebido em 26/Ago/2011

Aprovado em 31/Jan/2012 